\title{
A NOVEL DIFFERENTIALLY PUMPED UHV FLANGE IN THE CESR INTERACTION REGION*
}

\author{
Y.Li†, J.J.Cherwinka, Y.He and N.B.Mistry \\ Laboratory of Nuclear Studies, Cornell University, Ithaca, NY 14853 USA
}

\begin{abstract}
We describe the design and performance of a novel ultra-high vacuum flange used for the upgrade of the interaction region (IR) of the Cornell Electron Storage Ring (CESR) where the CLEO High Energy Experiment is located. The differentially pumped, Viton O-ring sealed flange requires a low sealing force, allowing remotely controlled mechanical engagement. This provides flexibility and improved safety for the existing CLEO Be-beam pipe and attached silicon vertex (SVX) detector during installation of the CESR IR UHV system. To ensure acceptable vacuum performance of the flange joint over the operational lifetime, the radiation degradation and the outgassing properties of the Viton Orings were investigated. We concluded that there was no significant seal degradation when the Viton O-rings were exposed to $5 \times 10^{6} \mathrm{Rad}$ of $\gamma$ radiation. The Viton O-ring outgassing rates were measured as a function of pumping time for various ambient air exposure times. Onedimensional finite element calculations showed that $\mathrm{O}$ ring outgassing would have virtually no impact on the UHV operation. The flange also forms an axial RF seal with a canted coil spring and a radial RF seal with a electric conductive O-ring. This allows the joint to carry image currents from a storage beam up to 1 Amp. The IR UHV system including this so-called 'magic' flange joint has been operated successfully for two years, and has been successfully engaged and disengaged twice for upgrades of the CLEO detector.
\end{abstract}

\section{INTRODUCTION}

To achieve higher luminosity at CESR/CLEO, an aggressive upgrade program (Phase III) has been well underway for two years. The upgrade program involves installation of a new HEP detector, CLEO-III, with improved particle identification and high trigger rate capability, and the installation of a pair of permanent magnet quadrupoles (PMQs) [1] and superconducting quadrupoles (SCQs) [2] positioned as close to the interaction point (IP) as possible to minimize beam size at the IP. Situated in the core of the CLEO detector and CESR IR is the double-walled thin beryllium vertex beam pipe (VBP) [3]. For installation flexibility and safety of the VBP and detector, it was very desirable to install the VBP with the CLEO central detector package (CDP), with minimum mass attached to the VBP. It is significantly more convenient to install the PMQs and SCQs without any vacuum chambers connected to the VBP after the
CDP installation. However, this installation scheme poses the challenge of making an ultra-high vacuum (UHV) compatible connection between the near IR vacuum system and the delicate VBP buried in the CDP, remotely through the warm-bore of the SCQ, as shown in Figure 1. In this paper, we describe the design of a novel UHV flange joint, the so-called 'magic' flange (MF), that makes the CESR/CLEO Phase III installation possible.

The CLEO-III detector with central VBP and vertex detector were installed in 1999, equipped with a MF to connect to the existing vacuum chamber through the existing normal quadrupoles. The remaining equipment, including PMQs and SCQs will be installed in July 2001.

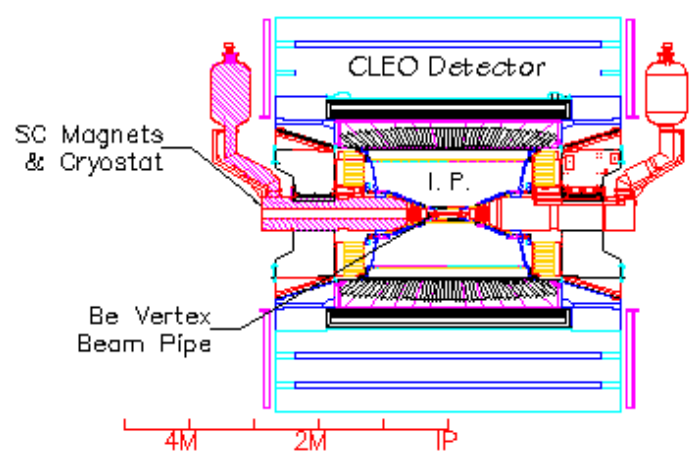

Figure 1. CESR Phase 3 IR, outlining the CLEO detector, vertex Be beam pipe and PMs \& SCQs.

\section{DESIGN}

\subsection{Design Considerations}

The following factors had to be considered in the design of the flange joint. First, it must be an UHV compatible joint, as the vacuum performance of the joint has a direct impact on the vacuum performance in the IR. The leak rate from atmosphere through the joint must be small compared to the very limited local pumping speed. Second, the joint must be able to engage and disengage reliably with low force to ensure the safety of the fragile vertex beam pipe at the IP without direct access. Furthermore, the joint must have adequate RF properties, so that (1) the higher-order mode (HOM) loss at the joint is small, and (2) there is no detectable RF leakage from the joint to affect the surrounding CLEO detector components. A design with a differentially pumped elastomer O-ring sealed joint was chosen.

\footnotetext{
* This work is supported by the National Science Foundation

†y167@ cornell.edu
} 


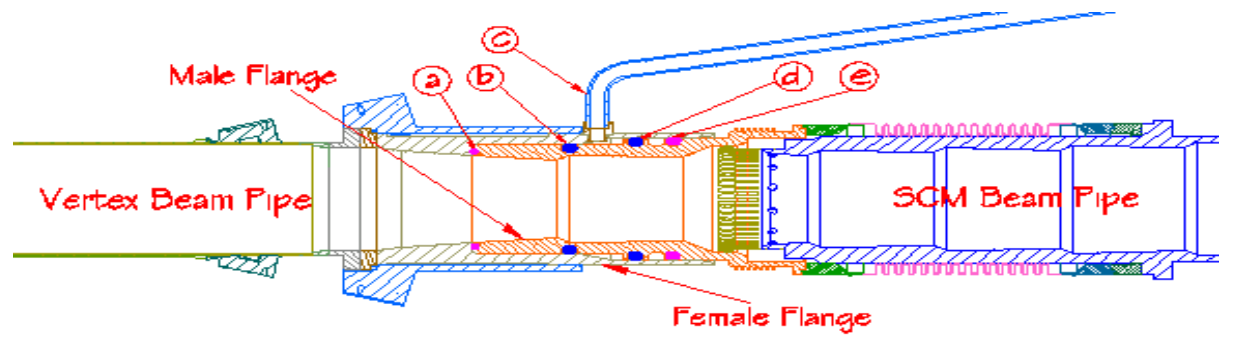

Figure 2. Design of the differentially pumped O-ring sealed 'magic' flange. The female flange (FMF) is attached to the Be vertex beam pipe, while the male flange (MMF) is incorporated onto the SCQ beam pipe [5]. (a) Be-Cu RFcontact spring; (b) inner Viton O-ring; (c) differential pumping line; (d) outer Viton O-ring; (e) EcE O-ring.

As shown in Figure 2, the design employed a double Viton ${ }^{\circledR}$ O-ring seal in a shaft-seal style to minimize the force required to form and disengage the seal, and to minimize the radial size of the joint.

\subsection{O-ring Thermal Outgassing}

For the elastomer seal, three types of gas loads need to be evaluated: leakage through the sealing surfaces, permeation through the O-ring and the outgassing from the O-ring. With the differential pumping, gas leakage and permeation are reduced and negligible. The thermal outgassing from the inner O-ring (see Figure 1), however, will contribute to the static pressure near the IP.

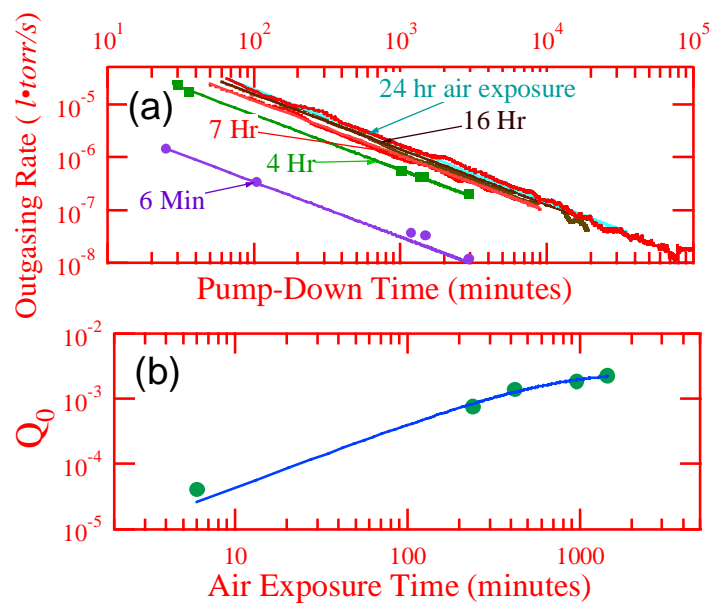

Figure 3. (a)Measured Viton O-ring outgassing rate as a function of pumping time with five air exposure times. (b)Fitting parameter $\mathrm{Q}_{0}$ vs. exposure time. The curve is the least $\chi^{2}$ fit to a function:

$$
Q_{0}=2.4 \times 10^{-3}\left(1-\exp \left(-t_{\text {exposure }} / 555.6\right)\right)
$$

Although the thermal outgassing of an O-ring may be significantly reduced through degassing in vacuum at elevated temperature, exposure of the O-rings to ambient air is inevitable during the installation. We measured the outgassing rate of the Viton O-rings used in the joint as a function of pumping time. The O-rings were first degassed in vacuum for 24 hours at $150^{\circ} \mathrm{C}$, then exposed to ambient air $\left(60 \%\right.$ relative humidity @ $\left.23^{\circ} \mathrm{C}\right)$ for various exposure times, and loaded into an outgassing measurement apparatus via a load-lock. Measured outgassing rates for the O-rings are shown in Figure 3a. The decrease of the outgassing rate with time is well represented by the function:

$$
\left.Q=Q_{0} / t_{\text {pumping }} \quad \text { (torr } \cdot \text { liter } / \mathrm{sec}\right)
$$

The fitting parameter $\mathrm{Q}_{0}$ depends on the air exposure time, as shown in Figure $3 \mathrm{~b}$. While the outgassing rate of a fully degassed O-ring increases rapidly initially with the exposure time, the increase levels off after $\sim 8 \mathrm{hr}$ air exposure. Since the installation time for both sides of the IR is certainly longer than 8 hours, we must take the saturated O-ring outgassing rate into account when evaluate the IR vacuum performance. As a matter of fact, the pressure profile for the CESR Phase III IR has been calculated [4], and the outgassing of the O-ring can be contained by the first titanium sublimation pump located at $\sim 3 \mathrm{~m}$ from the IP. Simulation [6] showed an acceptable beam-gas radiation background.

\subsection{O-ring Radiation Degradation}

Another important design issue is the effect of radiation on an elastomer. The most significant effect is the radiation hardening, or loss of resilience, of the O-ring, as the sealing performance may deteriorate from a hardened O-ring. We have measured the O-ring resilience loss, or the O-ring compression set (defined in Figure 4) as a function of $\gamma$ radiation dose, using an intense $\mathrm{Co}^{60}$ source at Ward Lab at Cornell. The results, given in Figure 5, showed a radiation induced compression set of $\leq 30 \%$ with $\gamma$ radiation dose up to $2 \times 10^{6} \mathrm{Rad}$, indicating acceptable sealing performance of the Viton O-ring for the expected service duration estimated from previous radiation measurements in the IP.

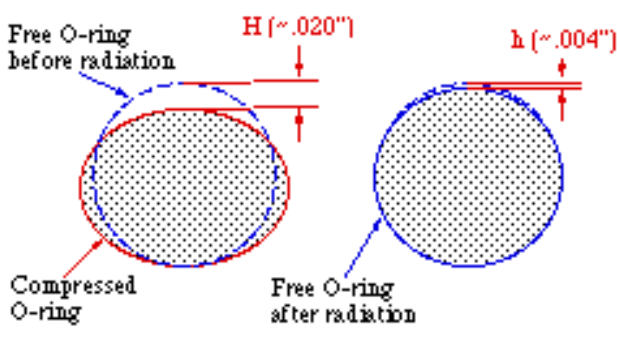

Radiation Compression Set $=\mathrm{h} / \mathrm{H} * 100$

Figure 4. Definition of Radiation Compression set. 


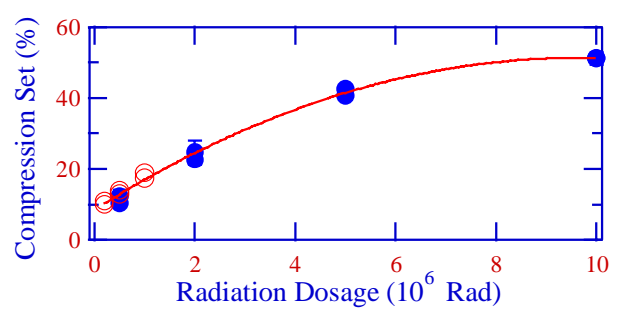

Figure 5. Viton O-ring compression set as a function of radiation dose. Open and solid circles are from two sets of measurements for six Viton O-rings.

\section{4. $R F$ seal/shield}

It is critical to minimize beam induced HOM loss at the flange joint for the safety of the flange joint, and to shield any RF leakage from affecting CLEO detector operation.

$\mathrm{A} \mathrm{Be}-\mathrm{Cu}$ spring (Bal Seal Inc.) is securely placed at the front of the male flange, as shown in Figure 2. The spring bridges any small gap between the front end of the male flange and a step in the female flange when the joint is formed. An electrically conductive elastomer (EcE) Oring (Laird Tech. Inc. EcE\#82, Ag-doped Fluorosilicone) is used to absorb any possible RF leakage from the joint. The RF seal/shield design had been confirmed to be adequate via bench measurement [7] with an RF network.

\subsection{Flange Engage and Disengage}

The flange engage/disengage mechanism is illustrated in Figure 6. As the SCQ pipe is manually inserted, the MMF on the SCQ beam pipe is guided into the FMF by tapered front end of the FMF. A worm-gear combination mounted on the FMF then turns a nut to thread the MMF into full engagement. The worm-gear is driven manually by a Nylon chain along an aluminum guiding rail that leads to an accessible location. UHV-compatible grease Fomblin ${ }^{\circledR}$ is applied to all O-rings to reduce the engaging force.

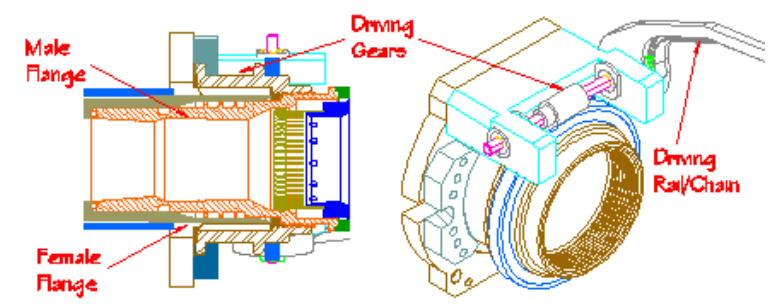

Figure 6. Magic flange engage and disengage mechanism.

\section{OPERATIONAL PERFORMANCE}

During the installation of the new CLEO III detector, the FMF was part of the new vertex Be beam pipe, and the MMF was incorporated into the modified existing Rare-Earth Quadrupole (REQ) beam pipe. The operation of the flange has proved to be very successful through two stages of CLEO detector installation. The engage and disengage mechanism operated very smoothly during the two installation procedures. A 45-liter/sec sputter-ion pump has been employed for the differential pumping. A direct comparison of the performance can be made between the IR UHV system before and after the installation of the magic flange and the new VPB, since there was no other modification to the remaining IR UHV system. The comparison of IR pressure data (Figure 7) between the two periods showed no negative impact of Viton thermal outgassing. The IR UHV system performance has been very satisfactory for over one year of operation.

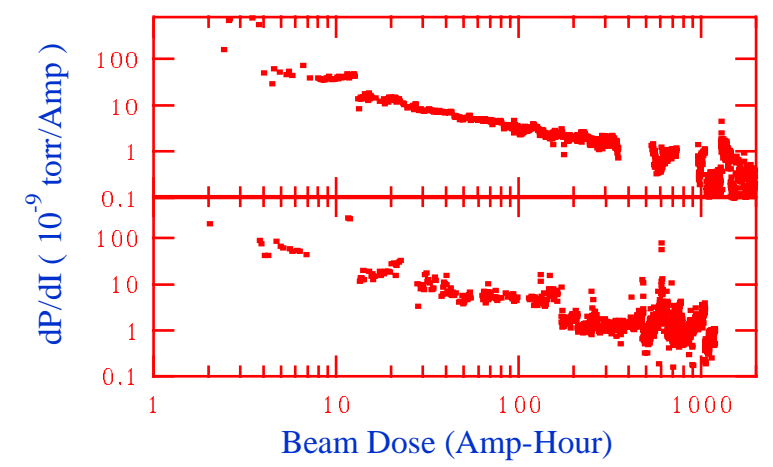

Figure 7. Comparison of CESR IR vacuum performance before (top trace, 1995-99) and after (bottom trace, 2000-2001) the installation of the magic flange, as monitored by a cold cathode gauge located $2.3 \mathrm{~m}$ from the IP.

\section{CONCLUSION}

A differentially pumped O-ring seal flange has been developed for the upgrade of CESR/CLEO. The implementation of the 'magic' flange greatly enhanced the safety and flexibility of the IR upgrade installation process.

\section{REFERENCES}

[1] W.Lou, D.Hartill, D.Rice, D.Rubin and J.Welch, Proc. 1997 Part. Accel. Conf., Vancouver BC, p.3237

[2] J.Welch, G.Dugan, E.Nordberg and D.Rice, Proc. 1997 Part. Accel. Conf., Vancouver BC, p.3383

[3] D.Cinabro, private communication

[4] R.Kersevan, Y.Li and N.Mistry, Proc. $\mathrm{e}^{+} \mathrm{e}^{-}$Factories '99, Tsukuba, Japan, p.242

[5] Y.He, Y.Li, N.Mistry and S.Greenwood, J. Vac. Sci. Technol. A19(4), 2001, in press

[6] S.Henderson, et al, Proc. 1999 Part. Accel. Conf., New York, NY, p.3221

[7] http://motor1.physics.wayne.edu/ cinabro/cinabro/ education/wsucure/reports/bursott.ps 